\title{
The Need for a Whole Systems Approach to Evidence Evaluation: An Update from the American College of Lifestyle Medicine
}

\author{
David L. Katz, MD, MPH, ${ }^{1,2}$ and Micaela C. Karlsen, $\mathrm{PhD}^{1,3}$
}

Editor's Note: In the course of developing this special issue, I learned of a relevant project through the Q\&A session at the end of a webinar on challenges of evaluating evidence from the American College of Lifestyle Medicine (ACLM). ACLM promotes multimodal care to improve health outcomes based on six identified components of lifestyle: diet, exercise, stress, sleep, social relations, and control of addictions. My follow-up led me to ACLM cofounder and long-time colleague David L. Katz, MD, MPH, and Micaela C. Karlsen, PhD, Director of the Lifestyle Medicine Economic Research Consortium. Katz informed me of an initiative of ACLM's to tackle the recurring challenge of how to appropriately evaluate evidence in lifestyle medicine. Although the initiative has not yet published any directive, Katz and Karlsen offered this background and commentary._John Weeks, Editor-in-Chief

$\mathbf{L}$ IFESTYLE MEDICINE IS A rapidly expanding discipline globally. ${ }^{1}$ As stated by Dr. Larry Green, Professor Emeritus in epidemiology and biostatistics at UCSF and former director of the federal Office of Health Promotion, "if we want more evidence-based practice, we need more practice-based evidence." Because lifestyle choices can have a major impact on mortality and morbidity, the importance of drawing evidence-based conclusions is of highest importance to inform practice recommendations. ${ }^{2}$

The concept of "whole systems research" was first proposed as a systems approach to evaluate complementary and alternative medical therapies that effect health or behavior in multiple domains. ${ }^{3}$ This idea was then developed in the context of evidence evaluation, and certain limitations of commonly accepted standards, such as the superiority of randomized controlled trials (RCTs), were discussed in the context of a holistic treatment of patients. ${ }^{4}$ The discussion has matured in recent years into active debate; with a critique on one side that practice recommendations in lifestyle medicine are inadequately supported by RCTs, ${ }^{5,6}$ and on the other voices calling attention to the contribution of other types of evidence. 7,8 $^{\text {at }}$

Models of holistic multimodal interdisciplinary care in which the entire model is deemed that "active ingredient" require assessment of the entire model of care versus other models. Reducing such a clinical approach to a study of, for example, fish oil capsules vs. a placebo restricts the question being asked to an absurd degree. Although the methods of randomized controlled trials can be used to evaluate effects of certain health behaviors, key limitations of RCTs include challenges in maintaining adherence over the course of the intervention, even greater challenges in adherence at followup, and the impracticality of continuing interventions beyond a few years at the longest, thus preventing the ability to address long-term health outcomes. ${ }^{9,10}$

In lifestyle medicine, our interests extend beyond the relatively short-term and isolated effects for which RCTs serve so well, to lifelong effects on vitality, and longevity for which they do not serve at all. In such contexts, RCTs can contribute some of the required "practice-based evidence," but the weight of evidence must be drawn from a variety of sources. The randomized controlled trial as the gold standard sells short the diverse subtleties of scientific truth and aggrandizes the RCT far beyond its desserts. When RCTs cannot serve as the primary evidence source, either because they have not been conducted, because the research question is not best answered in this study design, or because they may not be ethical, what are the alternative assemblies of evidence that can be used to assess causal relationships? Drawing from diverse evidence sources across methods, ${ }^{11-13}$ including bench science and observational epidemiology, as well as intervention research, is a

\footnotetext{
${ }^{1}$ American College of Lifestyle Medicine, St. Louis, Missouri.

${ }^{2}$ The True Health Initiative, Derby, Connecticut.

${ }^{3}$ Global Public Health and Applies Nutrition Programs, University of New England, Biddeford, Maine.
} 
practice aligned with best practices in epidemiology to identify causal relationships as described by the Bradford Hill criteria. ${ }^{14}$ Of note, the most critical element in Bradford Hill is temporality - confidence that the cause precedes the effect. This can be most easily demonstrated by RCTs but is not limited to RCTs, as prospective cohort studies also provide evidence of causality.

Science and evidence are conceptual tools in the service of truth and understanding, whereas methods to evaluate strength or weight of evidence for specific research questions are the concrete tools to reach truth. The tools must be appropriate for the application. To evaluate the tools available to appropriately distinguish between evidence-based lifestyle medicine interventions and unfounded claims, the American College of Lifestyle Medicine (ACLM) and the True Health Initiative recently convened a group of leading researchers in epidemiology and other health disciplines - to review strength of evidence (SOE) metrics and assess their application to the particular requirements of the lifestyle medicine domain. That project, titled Hierarchies of Evidence Applied to Lifestyle Medicine (HEALM), is now nearing completion. Informed by the systematic review results of SOE methods in current or recent use, HEALM will propose a new process for evaluating evidence that fills a gap for interventions or health behaviors relevant to long-term lifestyle and lifestyle medicine. The authors' intent is to offer this tool for other researchers to test and validate, thus informing strength of recommendation conclusions and lifestyle medical practice statements. Once published, the HEALM tool will be posted as a public resource on the ACLM and Lifestyle Medicine Economic Research Consortium websites for use, available to ACLM's growing membership base and also public website users.

\section{Author Disclosure Statement}

No competing financial interests exist.

\section{References}

1. Kushner RF, Sorensen KW. Lifestyle medicine: The future of chronic disease management. Curr Opin Endocrinol Diabetes Obes 2013;20:389-395.

2. Mokdad AH, Ballestros K, Echko M, et al. The state of US health, 1990-2016: burden of diseases, injuries, and risk factors among US states. JAMA 2018;319:1444-1472.
3. Ritenbaugh C, Verhoef M, Fleishman S, et al. Whole systems research: a discipline for studying complementary and alternative medicine. Altern Ther Health Med 2003;9: 32-36.

4. Verhoef MJ, Lewith G, Ritenbaugh C, et al. Complementary and alternative medicine whole systems research: beyond identification of inadequacies of the RCT. Complement Ther Med 2005;13:206-212.

5. Ioannidis JP. We need more randomized trials in nutritionpreferably large, long-term, and with negative results. Am J Clin Nutr 2016;103:1385-1386.

6. Rosen L, Manor O, Engelhard D, Zucker D. In defense of the randomized controlled trial for health promotion research. Am J Public Health 2006;96:1181-1186.

7. Willett WC. Diet and health-Finding a path to Veritas. Eur J Epidemiol 2018;33:127-135.

8. Barnard ND, Willett WC, Ding EL. The misuse of metaanalysis in nutrition research. JAMA 2017;318:1435-1436.

9. Feinstein AR, Horwitz RI. Problems in the "evidence" of “evidence-based medicine." Am J Med 1997;103:529535 .

10. Frieden TR. Evidence for health decision making-Beyond randomized, controlled trials. N Engl J Med 2017;377:465475 .

11. Nakamura R. Animal models and basic science-Bench to bedside: Session introduction. ILAR J 2011;52(Suppl_1): 493.

12. Hannan EL. Randomized clinical trials and observational studies: guidelines for assessing respective strengths and limitations. JACC Cardiovasc Interv 2008;1:211-217.

13. Sanson-Fisher RW, Bonevski B, Green LW, D'Este C. Limitations of the randomized controlled trial in evaluating population-based health interventions. Am J Prev Med 2007;33:155-161.

14. Fedak KM, Bernal A, Capshaw ZA, Gross S. Applying the Bradford Hill criteria in the 21st century: how data integration has changed causal inference in molecular epidemiology. Emerg Themes Epidemiol 2015;12:14.

Address correspondence to: Micaela C. Karlsen, PhD American College of Lifestyle Medicine St. Louis, $M O$ Chesterfield, MO 63006

E-mail: mkarlsen@lifestylemedicine.org 